\title{
Lack of interest in geriatrics among medical trainees a concern as population ages
}

\author{
n Cite as: CMAJ 2019 May 21;191:E570-1. doi: 10.1503/cmaj.109-5752
}

Posted on cmajnews.com on May 2, 2019.

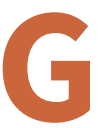
eriatricians are concerned by the lack of interest in geriatric medicine in this year's residency match. The specialty had the highest ratio of unfilled-to-filled spots, with 10 of 30 positions remaining vacant, according to the Canadian Resident Matching Service.

Dr. Phillip St. John, an associate professor of geriatrics at the University of Manitoba, said he hopes this year was an anomaly. "It's hopefully not another downward slip. The overall trajectory has been much more encouraging."

Since 2012, when only 11 of 26 spots were filled, the specialty has been growing steadily in trainee numbers. In both 2017 and 2018, there were 29 posts filled, out of 35 and 34 spots, respectively.

Dr. Samir Sinha, director of geriatrics at Sinai Health System, thinks that growth is partly due to pay reforms. Ontario, for instance, updated its fee schedule about a decade ago, bringing the pay of geriatricians up from about half that of internal medicine physicians to slightly below them. Many other provinces made similar remuneration changes, said Sinha. Growing awareness of the specialty, formed in the 1970 s, may also be helping, he said.

Still, we're well behind where we should be when it comes to the number of geriatricians needed to meet demand. There are just over 300 geriatricians working in Canada, with some provinces having only one, and the number of Canadians over 85 is expected to quadruple in the next two decades. Geriatric specialists say culture, medical training curricula and income all play a role in putting the specialty at a disadvantage.

"We're so focussed on anti-aging and youth that we don't actually tend to

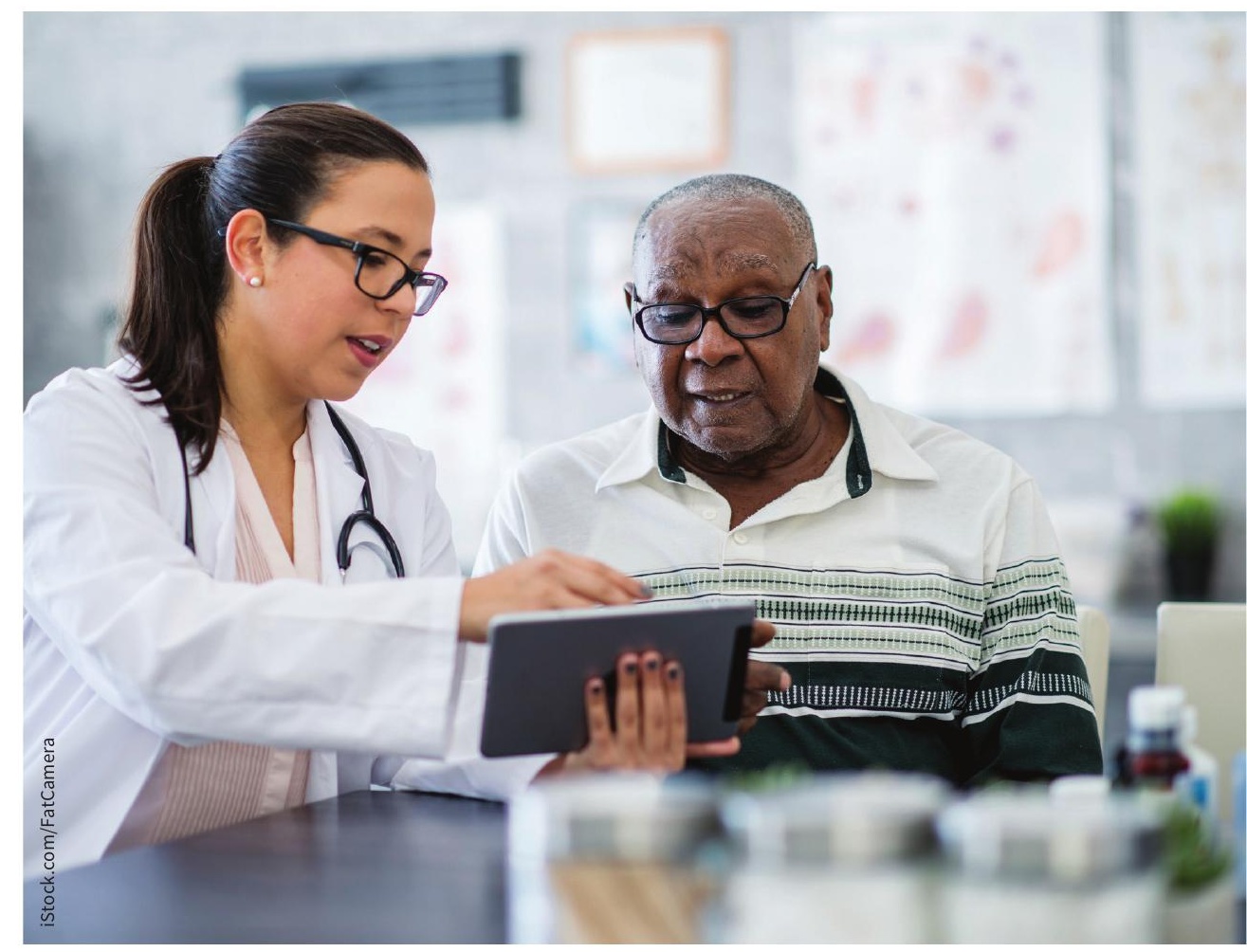

There are about 300 geriatricians in Canada, but some provinces have only one.

value older people in our society," said Sinha. Geriatrics relies on "a person's good will," given that other specialties with the same training length pay two or three times more.

But entrenched misperceptions don't help to foster good will, according to Dr. Nishi Varshney, a geriatrician and clinical instructor at the University of British Columbia, who says many physicians think the specialty is "depressing." Others "aren't fully aware of the expertise" that geriatricians possess.

Popular culture is also partially to blame, Varshney argued. She pointed to the book The House of God, well-read among medical students, in which doc- tors apply the term GOMER (get out of my emergency room) to older patients.

Yet studies show that geriatricians are among the most satisfied specialists. "It's quite a bit of fun," said St. John. Geriatricians have the luxury of spending more time with patients; consultations often surpass an hour. By improving quality of life in patients' later years, "we're not necessarily saving lives, but we almost always make them better," said St. John.

Varshney started the hashtag \#ADayInTheLifeOfAGeriatrician to highlight the positive aspects of the specialty. For instance, she appreciates that a substantial part of what she does is building 
the capacity of other providers to care for older patients.

Sinha thinks mandatory geriatric medicine rotations in medical school and internal medicine residencies would go a long way to rectifying the disconnect between the perception and reality of geriatric medicine.

"I have a good hunch that if you exposed people to clinical training ... the numbers would go up," he said. Currently, a four-week pediatric clerkship is mandatory in all medical schools, but a geriatric rotation is most often voluntary. "I think that many would be surprised to know that, in our taxpayer-funded system," Sinha said. Only 13 of 260 medical students at the University of Toronto did a geriatric medicine clerkship rotation last year, according to Sinha.

Varshney has seen first-hand how exposure to the speciality helps. When she works with medical students and residents, she often hears "that the emotions and the sentiments of why they did medicine in the first place, it comes back to them."

Unfortunately, making geriatric medicine a mandatory rotation isn't possible, currently, as there aren't enough teachers. Dr. Laura Diachun, chair of the Royal College of Physicians and Surgeons of Canada's specialty committee in geriatric medicine, pointed out that with so few geriatricians, many medical schools "couldn't possibly ensure that every medical student had at least one week of geriatrics."

In the meantime, some trainees have taken matters into their own hands. In 2011, Dr. Magda Lenartowicz, now a geriatrician in the United States, started the National Geriatrics Interest Group, which led to similar groups at medical schools across Canada. They hold extracurricular activities such as geriatric skills days and lectures.

But according to Varshney, medical schools should do a better job of prioritizing applicants who demonstrate an interest in care of older people in the first place. "Our society is an aging society, so we need to look at things from that frame of mind."

Wendy Glauser, Toronto, Ont. 